\title{
Berry Phase of Light under Bragg Reflection by Chiral Liquid-Crystal Media
}

\author{
Raouf Barboza, ${ }^{1, *}$ Umberto Bortolozzo, ${ }^{2}$ Marcel G. Clerc, ${ }^{1}$ and Stefania Residori ${ }^{2}$ \\ ${ }^{1}$ Departamento de Física, FCFM, Universidad de Chile, Casilla 487-3 Santiago, Chile \\ ${ }^{2}$ INLN, Université de Nice-Sophia Antipolis, CNRS, 1361 Route des Lucioles, 06560 Valbonne, France
}

(Received 29 December 2015; published 27 July 2016)

\begin{abstract}
A Berry phase is revealed for circularly polarized light when it is Bragg reflected by a chiral liquidcrystal medium of the same handedness. By using a chiral nematic layer we demonstrate that if the input plane of the layer is rotated with respect to a fixed reference frame, a geometric phase effect occurs for the circularly polarized light reflected by the periodic helical structure of the medium. Theory and numerical simulations are supported by an experimental observation, disclosing novel applications in the field of optical manipulation and fundamental optical phenomena.
\end{abstract}

DOI: 10.1103/PhysRevLett.117.053903

The Berry phase is a phenomenon well known to occur when dealing with cyclic adiabatic transformations [1]. Introduced by Berry for quantum systems undergoing a cyclic evolution under the action of a time-dependent Hamiltonian [1], the concept has been generalized to the accumulation of a geometric phase arising from topological aspects in different quantum and classical systems, among the most known are the Aharonov-Bohm effect and the precession of the oscillation plane of a Foucault pendulum (see, e.g., Ref. [2] for a review). A Berry phase for light is associated with the geometric manipulation of the polarization and/or the direction of propagation; see Ref. [3] and references therein. A geometric phase due to the polarization state manipulation was discovered by the pioneering work of Pancharatnam, who highlights it as an intrinsic property of polarized light [4]. Indeed, when the polarization of a beam describes a closed loop on the Poincare sphere, the final state differs from the initial one by a geometric phase, the Pancharatnam-Berry (PB) phase, proportional to the area enclosed by the loop. Based on this feature, space-variant wave plates have been specifically designed to realize PB phase optical elements with subwavelenght gratings, such as polarization sensitive diffraction gratings [5] and spiral phase elements [6], for laser radiation at $10.6 \mu \mathrm{m}$ wavelength. PB phase optical elements in the visible spectral domain have, then, been achieved by using nematic liquid crystals with patterned alignment, such as $q$ plates that generate helical modes of visible light $[7,8]$. Based on nematics, other PB phase optical devices, such as switchable lenses, beam splitters, and holographic elements, have been proposed [9] and, more recently, a diffractive lens with convergent (divergent) behavior depending on the left (right) handedness of the circular polarization of the input light has been realized $[10,11]$.

Here, we show that a Berry phase effect occurs for circularly polarized light when it is Bragg reflected by a chiral liquid-crystal medium of the same handedness. The optical wavelength and polarization handedness of the incident beam match, respectively, the Bragg wavelength and the handedness of the helical structure in the chiral medium. The geometric phase is introduced by the symmetry breaking in spin-momentum (handedness-direction) space and can be revealed by a space-variant optical axis of the uniaxial chiral layer. With the purpose of revealing the effect, we employ a chiral nematic liquid-crystal (CLC) cell with appropriately designed anchoring conditions at the entrance plane. Unlike conventional reflective elements, the meaningful component of the phase, here, is not introduced by optical path differences, but it results from the geometric phase accumulated at the input plane of the CLC cell. Indeed, if at the input plane the CLC layer is rotated with respect to a fixed reference frame, a geometric phase effect occurs during the reflection. By exploiting this property, and by introducing patterns of molecular anchoring at the entrance plane of the CLC cell, reflectors performing different wave front shaping can be designed and realized.

A qualitative comparison between a conventional mirror and a CLC medium is sketched in Fig. 1. At variance with the mirror [Fig. 1(a)], which reverses both the propagation direction and the polarization of the reflected light, the CLC cell [Fig. 1(b)] maintains the same polarization of the reflected beam. Indeed, CLCs are characterized by a self-assembled helical structure of their molecular arrangement and selective Bragg reflection: when light is circularly polarized with the same handedness of the chiral helix, the periodic helical structure leads to Bragg reflection at normal incidence whenever the optical wavelength $\lambda$ satisfies the condition $n_{o} P<\lambda<n_{e} P$, where $P$ is the chiral pitch and $\left\{n_{o}, n_{e}\right\}$ are the ordinary and extraordinary refractive indices, respectively [12]. The circularly polarized component with opposite handedness is transmitted through the chiral layer. These properties combined with the ability to change their pitch with electric or magnetic fields [13], with temperature [14] or light [15], make CLC attractive for applications such as dynamically controlled reflection or transmission filters [16], tunable lasers [17], 

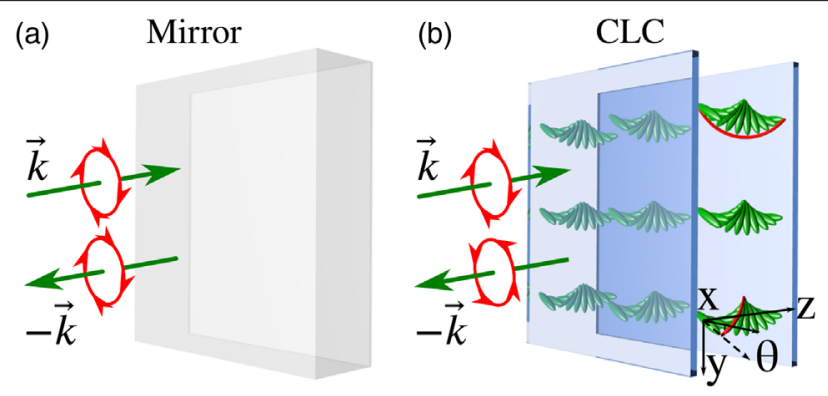

FIG. 1. Reflection from (a) a conventional mirror and (b) a chiral liquid-crystal (CLC) medium. The red arches depict the cholesteric helix; while the mirror reverses the polarization of the reflected light, a circularly polarized beam matching the handedness of the chiral helix maintains the same polarization when reflected.

and photoswitchable band gaps [18]. Here, we show that a Berry phase is always associated with the Bragg reflection at normal incidence, which allows adding novel functionalities to CLC layers, such as the ability to arbitrarily shape the reflected wave front.

A detailed explanation of such a geometric phase can be derived by considering a perfectly plane CLC layer with the director at its front face orientated at an angle $\theta$ with respect to the $x$ axis of a fixed reference frame $(x, y, z), \hat{z}$ coinciding with the axis of the chiral helix and with the propagation direction of the incident beam (see Fig. 1). We can now introduce a local coordinate system $\left(x^{\prime}, y^{\prime}, z\right)$, such that $\hat{x}^{\prime}$ is parallel to the molecular director at the input face of the CLC cell; that is, $\hat{x}^{\prime}=\hat{x} \cos \theta+\hat{y} \sin \theta$ and $\hat{y}^{\prime}=-\hat{x} \sin \theta+\hat{y} \cos \theta$. In the fixed reference frame a $\sigma$ handed circular polarization reads as $\hat{e}_{\sigma}=(\hat{x}+i \sigma \hat{y}) / \sqrt{2}$, which, in the local coordinate system, transforms to $\hat{e}_{\sigma}^{\prime}=\left(\hat{x}^{\prime}+i \sigma \hat{y}^{\prime}\right) / \sqrt{2}=e^{-i \sigma \theta} \hat{e}_{\sigma}$. Consider now a circularly polarized incident plane wave $\vec{E}_{i}=E_{i}(0) e^{i(k z-\omega t)} \hat{e}_{\sigma}$, with its wavelength inside the reflective band gap of the CLC, its polarization handedness matching that of the chiral helix, and at normal incidence. The incident wave is totally reflected by the CLC layer and its handedness is conserved after the reflection. In the fixed reference frame the reflected wave can, thus, be written as $\vec{E}_{r}=$ $E_{r}(0) e^{-i(k z+\omega t)} \hat{e}_{-\sigma}$. If the axis of the CLC layer is chosen to coincide with $\hat{x}(\theta=0)$, we can apply the well-known relation $E_{r}(0)=r E_{i}(0)$, with $r$ the reflection coefficient [13]. However, if the CLC is rotated by an angle $\theta$ with respect to $\hat{x}$, the relation between the amplitude of the incident and the reflected electric field must be considered in the local coordinate system and the phase arising from the coordinate transform from the local to the fixed reference must be taken into account; they do not necessarily cancel out. Therefore, the incident and the reflected beam, respectively, $\vec{E}_{i}=E_{i} \hat{e}_{\sigma}$ and $\vec{E}_{r}=E_{r} \hat{e}_{-\sigma}$, in the local coordinate system read as $\vec{E}_{i}=E_{i}^{\prime} \hat{e}_{\sigma}^{\prime}=E_{i}^{\prime} e^{-i \sigma \theta} \hat{e}_{\sigma}$ and $\vec{E}_{r}=E_{r}^{\prime} \hat{e}_{-\sigma}^{\prime}=$ $E_{r}^{\prime} e^{i \sigma \theta} \hat{e}_{-\sigma}$, respectively. The explicit dependence on $z$ and $t$ is dropped for the sake of clarity. As a consequence, the reflection coefficient is given by $r=E_{r}(0) / E_{i}(0)=$ $e^{2 i \sigma \theta} E_{r}^{\prime}(0) / E_{i}^{\prime}(0)$, which can also be written as $r=r^{\prime} e^{i \Phi_{B}}$, where $\Phi_{B}=2 \sigma \theta$ is the Berry phase, $r^{\prime}$ the reflection coefficient in the local coordinate and does not depend on $\theta$. Note that $\Phi_{B}$ has a pure geometric origin, that is, it does not originate from an extra optical path but from a purely geometrical transformation, namely, the flipping of the propagation direction while maintaining the same helicity of the circular polarization, and the rigid rotation of the CLC layer. Based on the same analysis, it is easy to show that the net geometric phase effect is canceled for the transmitted component (case of thin layers). Indeed, in such case the amplitude of the transmitted wave can be written as $\vec{E}_{t}=E_{t} e^{i(k z-\omega t)} \hat{e}_{\sigma}$, which transforms in the local coordinate system as the incident beam, i.e., $\vec{E}_{t}=E_{t}^{\prime} \hat{e}_{\sigma}^{\prime}=E_{t}^{\prime} e^{i \sigma \theta} \hat{e}_{\sigma}$, and, correspondingly, the transmission coefficient $t=E_{t}(L) / E_{i}(0)=E_{t}^{\prime}(L) / E_{i}^{\prime}(0)$; thus, $t=t^{\prime}, t^{\prime}$ is the transmission coefficient in the local reference, independent from $\theta$.

The dependence of the reflection coefficient on the molecular angle $\theta$ at the input plane of the CLC layer can be derived analytically from the light propagation equations and by using the coupled mode theory. This treatment can be found in the Supplemental Material [19] with the included references [20-22]. As a result, all the possible cases can be simply summarized in a scattering matrix, which reads as

$$
\left[\begin{array}{c}
E_{\sigma}(L) \\
E_{-\sigma}(0)
\end{array}\right]=\left[\begin{array}{cc}
t & r^{\prime} e^{-2 i \sigma \theta_{L}} \\
r^{\prime} e^{2 i \sigma \theta_{0}} & t
\end{array}\right]\left[\begin{array}{c}
E_{\sigma}(0) \\
E_{-\sigma}(L)
\end{array}\right],
$$

where $E_{\sigma}(0)$ is a $\sigma$-handed circularly polarized wave incident on the front face of the CLC layer and $E_{-\sigma}(L)$ is a circularly polarized wave with the same handedness but propagating in the opposite direction and incident on the end face of the CLC layer (cf. Fig. 2). The output fields $E_{\sigma}(0)$ and $E_{\sigma}(L)$ are each built by two contributions: the beam transmitted through the CLC layer from the other end of the cell and the beam propagating from the same side and Bragg reflected by the periodic helix in the CLC layer. From the scattering matrix terms it can be seen that the reflection coefficients are accompanied by a geometric phase, respectively, $\Phi_{B 0}=2 \sigma \theta_{0}$ and $\Phi_{B L}=-2 \sigma \theta_{L}$, where the angles $\theta_{0}$ and $\theta_{L}$ denote the anchoring direction of the molecules at the entrance, respectively, exit plane of the CLC layer. The derived scattering matrix Eq. (1) is similar to those used to describe a transmissive and reflective anisotropic uniaxial geometric phase element; see Ref. [23] and references therein. However, rather than linking the reflected or the transmitted light to the incident field, it connects circularly polarized components of reflected and transmitted waves to the forward and the backward propagating circularly polarized incident fields, where 


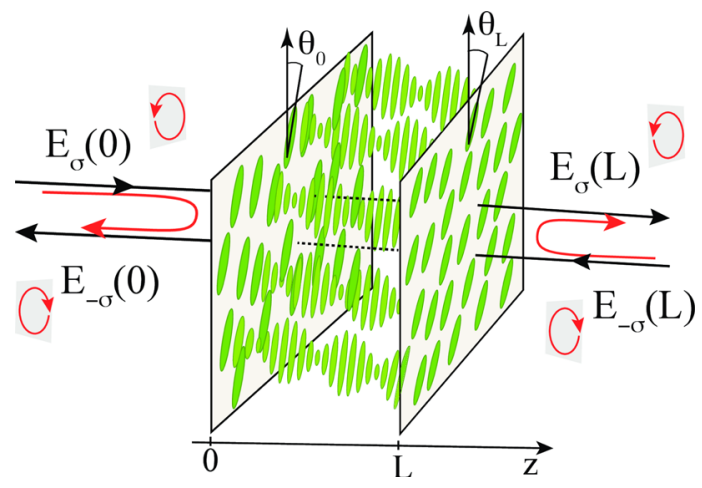

FIG. 2. Modes of light propagation in the CLC layer. Only the Bragg regime is considered. The (red) curved arrows indicate the Bragg-reflected beams to which a Berry phase is associated.

the handedness considered is that of the cholesteric cell. Moreover, unlike for the untwisted structures, the phase of the off-diagonal terms (that carry the information about the geometric phase) can be made independent with dissimilar anchoring angle at the faces of the geometric phase element. A scheme representing the scattering processes is displayed in Fig. 2.

Numerical simulations were performed with the finitedifference time-domain method [24], using a freely available software package [25]. We have calculated the geometric phase, taking the phase of the reflection coefficient of a lefthanded $(\sigma=+1)$ circularly polarized beam by a left handed helix and, a right handed $(\sigma=-1)$ circularly polarized beam by a right handed helix [19]. The results are shown in Fig. 3, where $\Phi_{B}$ is plotted versus $\theta$ the anchoring angle at the input of the cell. The right- and the left-handed case were considered. The geometric phase varies linearly and the slope depends on the handedness of the polarization. The expected dependence $\Phi_{B}(\theta)=2 \sigma \theta$ can be clearly verified.

To experimentally reveal the effect, we have set up a Michelson-type interferometer in which one of the mirrors is substituted by a CLC cell, as depicted in Fig 4. The cell is

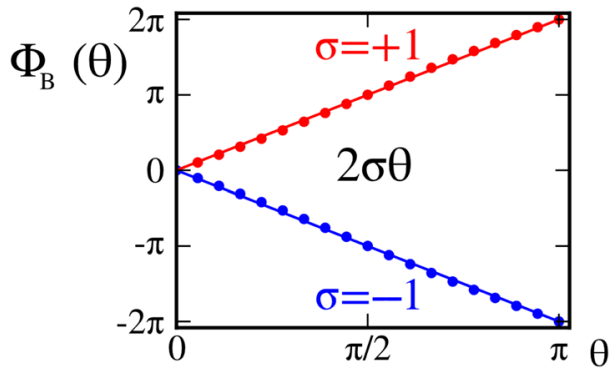

FIG. 3. Numerically calculated Berry phase versus the angle $\theta$ of the molecular alignment at the input plane of the CLC cell. The results (dots) match their corresponding (lines) analytical prediction $\Phi_{B}(\theta)=2 \sigma \theta$, where $\sigma= \pm 1$ is the handedness of the cholesteric helix and of the input polarization. filled with a CLC mixture composed of the nematic E7 $\left(n_{e}=1.7472\right.$ and $n_{o}=1.5217$ at $589.3 \mathrm{~nm}$ at $\left.20^{\circ}\right)$ doped with the chiral agent CB15 (41\% in weight). The chiral mixture is injected between two planar rubbed polyvinylalcohol-coated glass plates with $L=9 \mu \mathrm{m}$ thick spacers. The front plane of the cell has been rubbed mechanically in two steps. After rubbing the first half of the glass plate, the second half is rubbed in a direction orthogonal to the previous one. This procedure provides us with a cell with two regions of orthogonal planar alignment, the anchoring angle being $\theta_{\mathrm{I}}=0$ and $\theta_{\mathrm{II}}=\pi / 2$ in each region, respectively (see Fig 4). The helical structure of the chiral mixture is perpendicular to the confining walls and has a pitch $P$ such that $\sim 50$ half-pitches are contained along the cell thickness. Note that the cell can be considered thick insofar as just a few pitches of the helix close to the entrance plane are enough to provide the total construction of the Braggreflected beam. The incident beam is circularly polarized with the same handedness of the chiral layer and overlaps the two regions of orthogonal anchoring. Because of the orthogonal anchoring conditions, the two regions of the CLC cell induce a geometric phase shift on the reflected wave front. The phase shift is revealed by interfering the probe with the reference wave, which is reflected by an ordinary mirror. A quarter-wave plate is used to convert the probe to a circularly polarized wave with the same handedness as the CLC helix. Another quarter-wave plate is inserted in the reference arm in order to maximize the visibility of the fringes in the interference pattern, which is recorded by a CCD camera (cf. Fig. 4). Figure 5(a) displays an instantaneous snapshot of the CLC cell, showing the interface between regions I and II. An experimentally recorded interference pattern is shown in Fig. 5(b). Because the probe beam overlaps two regions with different orientation of the optical axis, a phase shift is expected to appear in the fringe interference pattern. Indeed, dislocation

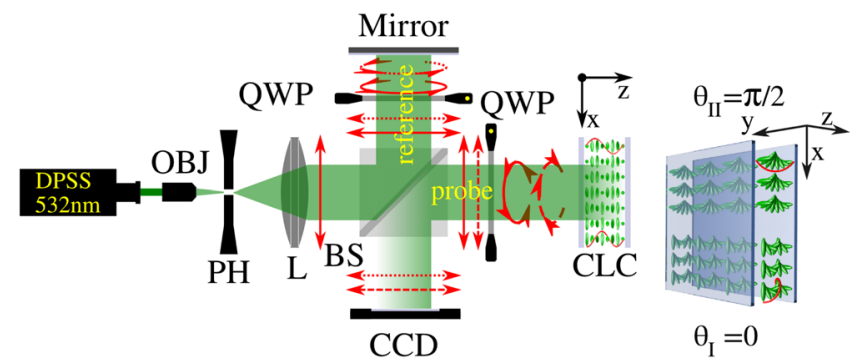

FIG. 4. Experimental setup. A diode pumped solid-state (DPSS) laser at $532 \mathrm{~nm}$ is divided into a reference and a probe beam by the beam splitter (BS), then recombined in a Michelsontype interferometer onto a CCD camera; while the reference is reflected by an ordinary mirror, the probe beam is reflected by a CLC cell that has two regions of planar alignment in its entrance plane (anchoring angles $\theta_{\mathrm{I}}=0$ and $\theta_{\mathrm{II}}=\pi / 2$ ), as shown in the inset. The red helical arches depict the cholesteric helix for a better clarity on the handedness. OBJ, objective; $\mathrm{PH}$, pinhole; L, lens; QWP, quarter-wave plate. 


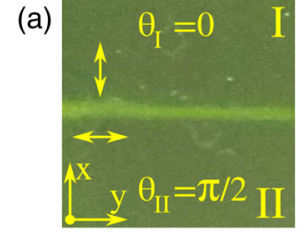

(b)

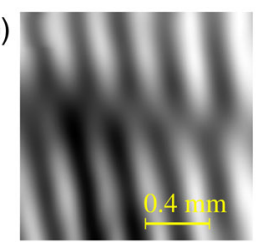

(c)

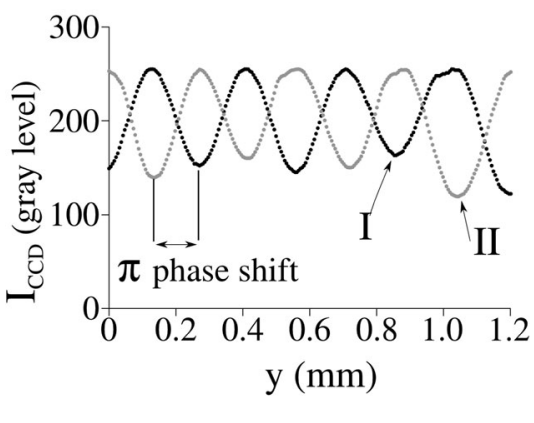

FIG. 5. (a) Instantaneous snapshot of the CLC cell showing the interface between regions I and II corresponding to the orthogonal anchoring $\theta_{I}=0$ and $\theta_{I I}=\pi / 2$. (b) Interference pattern recorded for a probe beam hitting the cell across the interface; dislocation lines evidence the $\pi$ phase shift between regions I and II. (c) One-dimensional intensity profiles taken on the interference pattern in regions I and II, respectively.

lines can clearly be distinguished across the interface, evidencing the $\pi$ phase difference between regions I and II, corresponding to $\Phi_{B}=2 \sigma \theta=\pi$, with $\sigma=1$ and $\theta=\pi / 2$ in this experiment. Figure 5(c) shows two one-dimensional intensity profiles taken on the fringe interference pattern in regions I and II, respectively. Again, the geometric phase shift can be clearly appreciated.

In conclusion, we have demonstrated that a Berry phase exists whenever circularly polarized light is Bragg reflected by a CLC layer. Such a geometric phase originates from the orientation of the chiral layer at the entrance plane of the cell. By using more advanced alignment techniques based on photopolymers [26] and holographic recording $[10,11]$ or direct laser writing $[10,27]$, a nearly arbitrary profile of planar anchoring can be achieved. Arbitrary profiles of a geometric phase can then be achieved with application in complex wave front shaping without the use of backreflectors. A recent application was demonstrated by Kobashi, Hiroyuki, and Masanori [28]; however, reference to geometric phase was not mentioned. Moreover, operation in different spectral regions could easily be achieved by changing the reflective band gap of the CLC layer, and broadband devices could be obtained [29] by using the multitwisted layer architecture [30].

R. B. acknowledges FONDECYT POSTDOCTORADO 3140577 for financial support. M. G.C. thanks FOND ECYT 1150507 for financial support.

*raouf.barboza@ing.uchile.cl

[1] M. V. Berry, Proc. R. Soc. A 392, 45 (1984).
[2] F. De Zela, Theoretical Concepts of Quantum Mechanics, edited by M. R. Pahlavani (InTech, 2012).

[3] K. Y. Bliokh, Y. Gorodetski, V. Kleiner, and E. Hasman, Phys. Rev. Lett. 101, 030404 (2008).

[4] S. Pancharatnam, Proc. Indian Acad. Sci. A 44, 247 (1956).

[5] Z. Bomzon, G. Biener, V. Kleiner, and E. Hasman, Opt. Lett. 27, 1141 (2002).

[6] G. Biener, A. Niv, V. Kleiner, and E. Hasman, Opt. Lett. 27, 1875 (2002).

[7] L. Marrucci, C. Manzo, and D. Paparo, Phys. Rev. Lett. 96, 163905 (2006).

[8] S. R. Nersisyan, N. V. Tabiryan, D. Mawet, and E. Serabyn, Opt. Express 21, 8205 (2013).

[9] L. Marrucci, C. Manzo, and D. Paparo, Appl. Phys. Lett. 88, 221102 (2006).

[10] J. Kim, Y. Li, M. N. Miskiewicz, C. Oh, M. W. Kudenov, and M. J. Escuti, Optica 2, 958 (2015).

[11] A. M. W. Tam, F. Fan, H. S. Chen, D. Tao, V. Chigrinov, H. S. Kwok, and Y. S. Lin, SID Symp. Digest Tech. Papers 46, 8 (2015).

[12] P. G. de Gennes and J. Prost, in The Physics of Liquid Crystals, International Series of Monographs on Physics (Clarendon Press, Oxford, 1995).

[13] P. Yeh and C. Gu, in Optics of Liquid Crystal Displays, Wiley Series in Pure and Applied Optics (Wiley, New York, 1999).

[14] S.-Y. Tzeng, C.-N. Chen, and Y. Tzeng, Liq. Cryst. 37, 1221 (2010).

[15] D. Wei, A. Iljin, Z. Cai, S. Residori, and U. Bortolozzo, Opt. Lett. 37, 734 (2012).

[16] M. Mitov and N. Dessaud, Nat. Mater. 5, 361 (2006).

[17] H. Coles and S. Morris, Nat. Photonics 4, 676 (2010).

[18] U. A. Hrozhyk, S. V. Serak, N. V. Tabiryan, T. J. White, and T. J. Bunning, Opt. Express 18, 9651 (2010).

[19] See Supplemental Material at http://link.aps.org/ supplemental/10.1103/PhysRevLett.117.053903 for details regarding the scattering matrix and the numerical simulations.

[20] P. Oswald and P. Pieranski, in Nematic and Cholesteric Liquid Crystals: Concepts and Physical Properties Illustrated by Experiments, Liquid Crystals Book Series (CRC Press, Boca Raton, FL, 2005).

[21] V. A. Belyakov, in Diffraction Optics of ComplexStructured Periodic Media, Partially Ordered Systems (Springer, New York, 1992).

[22] J. Joannopoulos, S. Johnson, J. Winn, and R. Meade, Photonic Crystals: Molding the Flow of Light, 2nd ed. (Princeton University Press, Princeton, NJ, 2011).

[23] K. Y. Bliokh, F. J. Rodriguez-Fortuno, F. Nori, and A. V. Zayats, Nat. Photonics 9, 796 (2015).

[24] A. Taflove and S. Hagness, in Computational Electrodynamics: The Finite-Difference Time-Domain Method, 3rd ed., Artech House Antennas and Propagation Library (Artech House, Norwood, 2005).

[25] A. F. Oskooi, D. Roundy, M. Ibanescu, P. Bermel, J. D. Joannopoulos, and S. G. Johnson, Comput. Phys. Commun. 181, 687 (2010). 
[26] V. G. Chigrinov, V. M. Kozenkov, and H.-S. Kwok, in Photoalignment of Liquid Crystalline Materials: Physics and Applications, Wiley-SID Series in Display Technology (John Wiley \& Sons, Ltd., Chichester, 2008).

[27] M. N. Miskiewicz and M. J. Escuti, Opt. Express 22, 12691 (2014).
[28] Kobashi Junji, Yoshida Hiroyuki, and Ozaki Masanori, Nat. Photonics 10, 389 (2016).

[29] Y. Li, J. Kim, and M. J. Escuti, Proc. SPIE Int. Soc. Opt. Eng. 8274, 827415 (2012).

[30] R. K. Komanduri, K. F. Lawler, and M. J. Escuti, Opt. Express 21, 404 (2013). 